Harold A. McAlister and William I. Hartkopf (eds.)

\title{
Visual Binary Orbit Calculation with the Help of a PC: One Possible approach
}

\author{
Z. CATOVIĆ \\ Math. Fac. Studentski trg 16, 11000 Beograd, Yugoslavia \\ D. OLEVIĆ \\ Astron. Obs., Volgina 7, 11050 Beograd, Yugoslavia
}

\begin{abstract}
In this work we suggest one possible approach to "computerization" of the process of the visual double star orbit determination. Our computer program, based on the original method (Olevic 1989), is interactive which means that the calculator of the orbit makes the final decision about the choice of the best orbit.
\end{abstract}

\section{INTRODUCTION}

The theoretical base for the program is the linear method of least squares (MLS). In the first, geometrical part of the program, an elliptical orbit is determined by MLS applied on the observations plus one "falsified observation" (FO) chosen by the calculator. Conditional equations are from the equation of conical section in the rectangular coordinates, where all of the elements of the orbit appear as linear parameters. The orbit should pass throught FO and fit the regular observations by the least squares principle. Therefore, the choice of the position of FO is a crucial point in the program. That position should be chosen so that the solution of the applied MLS is an ellipse. By changing the position of FO one can draw a large number of elliptical orbits and analyse them.

In the second, dynamical part of the program, the mean motion and time of periastron passage are determined by employing MLS again; this time conditional equations result from the connection between mean anomaly and time, which is actually Kepler's equation. In this part of the program, one can also check the viability of Kepler's second law by employing the routine for calculation of the elliptical sector areas.

\section{GMLS \& DMLS}

We designate the geometrical part of the program described above as GMLS and the dynamical part as DMLS. GMLS coefficients $Z_{1}, \ldots, Z_{5}$ are to be determined from the conditional equations:

$$
Z_{1} X_{i}^{2}+Z_{2} Y_{i}^{2}+2 Z_{3} Y_{i} X_{i}+2 Z_{4} X_{i}+2 Z_{5} Y_{i}+1=0 ; \quad i=1, \ldots, N .
$$

where $N$ is the number of observations. It is clear that the conical section obtained by GMLS could not be an ellipse in the general case. Therefore, we should put FO on that position from which the influence of FO on the GMLS should be on towards an elliptical orbit. A good starting point for this search (in most of the cases, but not all) could be a half of the unobserved arc, with the mean distance (calculated for the observations) from the primary. If we demand that the conical section which is to be determined pass through FO with coordinates $\left(X_{f}, Y_{f}\right)$, we can reduce the number of degrees of freedom from 
5 to 4 . In our case, we chose coefficient $Z_{3}$ to be determined directly, from the condition that the solution should pass through $\left(X_{f}, Y_{f}\right)$. Therefore new conditional equations are:

$$
\begin{array}{r}
Z_{1}\left[X_{i}\left(X_{i}-\frac{X_{f}}{Y_{f}} Y_{i}\right)\right]+Z_{2}\left[Y_{i}\left(Y_{i}-\frac{Y_{f}}{X_{f}} X_{i}\right)\right]+Z_{4}\left[2 X_{i}\left(1-\frac{X_{i}}{X_{f}}\right)\right]+ \\
Z_{5}\left[2 Y_{i}\left(1-\frac{Y_{i}}{Y_{f}}\right)\right]+\left(1-\frac{X_{i} Y_{i}}{X_{f} Y_{f}}\right)=0 ; i=1, \ldots, N
\end{array}
$$

and $Z_{3}$ directly from:

$$
Z_{3}=-Z_{1}\left(\frac{X_{f}}{2 Y_{f}}\right)-Z_{2}\left(\frac{Y_{f}}{2 X_{f}}\right)-Z_{4}\left(\frac{1}{Y_{f}}\right)-Z_{5}\left(\frac{1}{X_{f}}\right)-\left(\frac{1}{2 X_{f} Y_{f}}\right) .
$$

On the other hand, if we demand that conical section passes close to but not exactly throught FO (by giving large weight to the FO) then the conditional equations are the equations of type (1).

Depending on the position of FO, the solution obtained might not necessarily be an ellipse that is automatically detected by the program. In that case further calculations are canceled and the program turns to the new choice of FO or exits. However if the orbit obtained is the elliptical one, then orbital elements are derived by Kovalsky's formulas (from $Z_{1}, \ldots, Z_{5}$ ) (Aitken 1963) and $n$ and $\tau$ are determined by the DMLS. Conditional equations of the dynamical part of the program are:

$$
n t_{i}-\beta=M_{i} ; \quad i=1, \ldots, N,
$$

where $\beta=n \tau ; \quad M_{i}=E_{i}-e_{i} \sin E_{i}$. Mean anomalies $M_{i}$ associated with the times of observation $t_{i}$ are calculated with the help of orbital elements obtained by GMLS. However, since the original observations (observed apparent places) are not placed exactly on the apparent orbit (calculated by GMLS), neither will their projections on the orbital plane be placed on the true orbit exactly. Therefore, by the above treatment we actually associate apparent positions to their counterparts on the apparent orbit which are placed on the intersection of the apparent orbit and line drawn from primary $A$ to the apparent place. That point-intersection is projected on the true orbit (with elements obtained by GMLS) in order to evaluate eccentric anomalies associated with the time of observation. At this point we will just note that the problem of "association of anomaly to the time" could be solved in a different way also (for example: minimal distance from $B$ to the apparent orbit).

\section{PRACTICAL EXAMPLE}

We took, for example, the double star ADS 1227, whose orbit has been taken from Couteau (1981). Three orbits are calculated. They are shown in Figure 1, with coordinates of FO's by which they are calculated, and corresponding orbital 

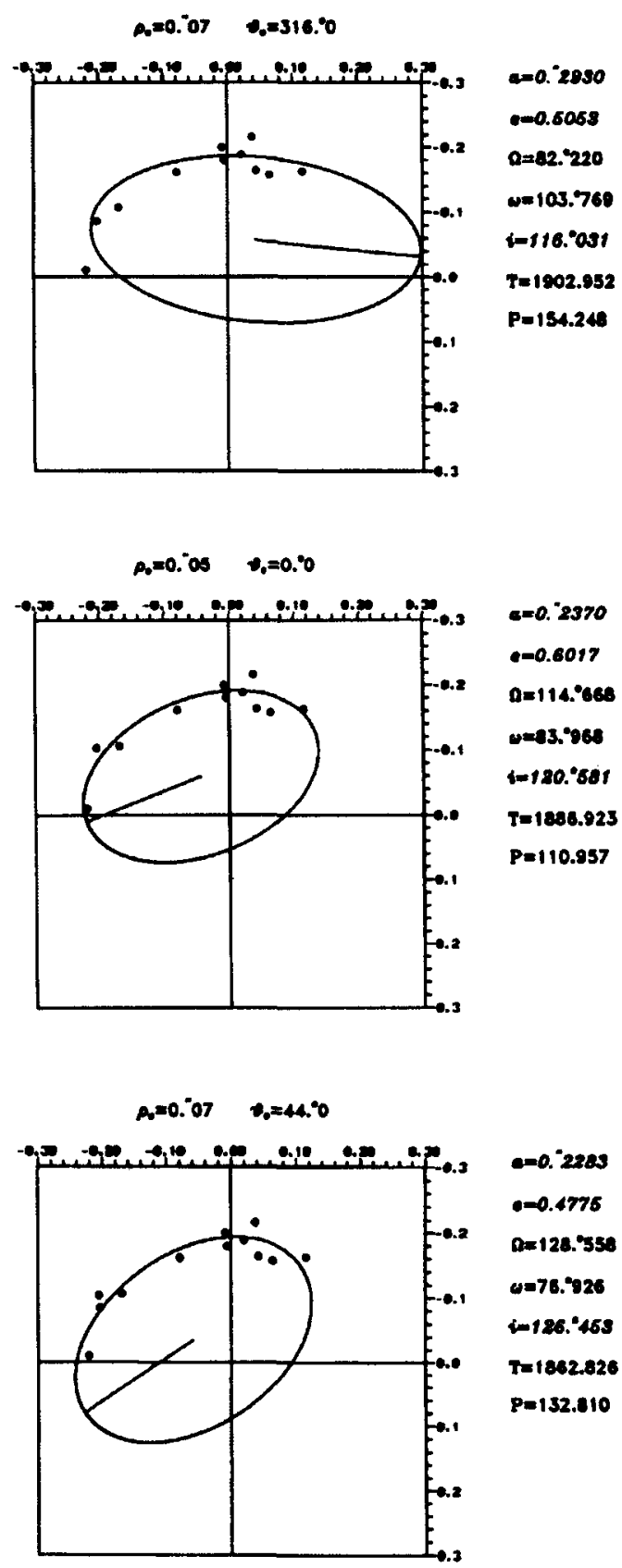

elements. Here we are not presenting $(O-C)$ because of space limitations, but detailed analysis will be published elsewhere. GMLSQ conditional equation for calculation in this case was equation (1).

\section{CONCLUSION}

The introduction of FO appears to us of twofold interest for future work. First, from a theoretical point of view, the introduction of FO opens many questions: in which area are FO's which provide elliptical solutions, or is it possible to connect dynamical conditions (Kepler's second law) with choice of FO? Second, from a practical point of view, the simple application software we have developed could be improved in many ways (for instance by adding a routine for differential corrections). Finally we note that, in the Astronomical Observatory in Beograd, we have calculated seven orbits of visual binaries, with the help of the program presented here, which have been published in the IAU Commision 26 Circulars.

\section{REFERENCES}

Aitken, R.G. 1963, Double Stars, New York

Couteau, P. 1978, L'Observation Des Etolies Doubles Visuelles, Flammarion

Olevic, D. 1989, private communication

FIGURE 1. Three calculated orbits for ADS 1227 